\title{
Approximate reduction of non-linear discrete models with two time scales
}

\author{
L. Sanz , R. Bravo de la Parra and E. Sánchez \\ Departmento Matemática Aplicada, E.T.S. Ingenieros Industriales, c. José Gutiérrez Abascal 2, \\ 28006 Madrid, Spain; Departmento Matemáticas, Universidad de Alcalá, 28871 Alcalá de \\ Henares, Spain; UR GEODES, IRD, 32, Avenue Henri Varagnat, 93143 Bondy cedex, France
}

\begin{abstract}
The aim of this work is to present a general class of nonlinear discrete time models with two time scales whose dynamics is susceptible of being approached by means of a reduced system. The reduction process is included in the so-called approximate aggregation of variables methods which consist of describing the dynamics of a complex system involving many coupled variables through the dynamics of a reduced system formulated in terms of a few global variables. For the time unit of the discrete system we use that of the slow dynamics and assume that fast dynamics acts a large number of times during it. After introducing a general two-time scales nonlinear discrete model we present its reduced accompanying model and the relationships between them. The main result proves that certain asymptotic behaviours, hyperbolic asymptotically stable (A.S.) periodic solutions, to the aggregated system entail that to the original system.
\end{abstract}

Keywords: nonlinear discrete models; aggregation of variables; time scales; population dynamics

\section{Introduction}

Ecological models always entail a decision on the level of detail to be included in them. This decision should be taken on the basis of optimizing the profit of the study. Any model is a compromise between generality and simplicity on the one hand and biological realism on the other. The more biological details are included in specifying a model, the more complicated and specialized it becomes. Models describing ecological systems in detail involve a very large number of coupled variables, which implies that the only methods to study them are computer simulations. At the other extreme, very simple models, which are mathematically tractable, do not justify how much have to do with the real systems they are supposed to represent.

Nature offers many examples of systems where several processes act at different time scales. It is then usual to consider those events occurring at the fastest scale as being instantaneous with respect to the slower ones. This sort of decoupling implies a reduction of the number of variables or parameters needed to describe the evolution of the system. A subsequent issue is to determine conditions for these reductions to give good approximations of the real results. An example of this general framework are the so-called 
aggregation methods which study the relationship between a large class of two time scales complex systems and their corresponding aggregated or reduced systems. The aim of aggregation methods is twofold: on the one hand they construct the reduced systems that summarize the dynamics of the complex ones, thus simplifying their analytical study and on the other hand, looking at the relationship in the opposite sense, the complex systems serve as explanations of the simple form of the aggregated ones.

The study of these methods was initiated about fifteen years ago by Auger , in the frame of ordinary differential equations. The main effort spent in deriving the so-called aggregated systems and a general formal computational method, the so-called quick derivation method, was described by Auger in a large class of systems possessing one or several invariants. The method was refined and justified in terms of perturbation techniques and in the application of an adequate version of the center manifold theorem [8], see Auger and Roussarie

In Bravo de la Parra e and Sánchez the case of linear, density independent, time discrete systems is studied; a very general linear model with two time scales is aggregated and it is proved that the elements defining the asymptotic behaviour of the general and the aggregated systems are equal up to a certain order. These results are applied to models of structured populations with subpopulations in each stage class associated to different spatial patches or individual activities, considering a fast time scale for patch or activity dynamics and a slow time scale for the demographic process. The results for the case where the time unit of the system is chosen to be that of the slow dynamics are generalized in Sanz and Bravo de la Parra assuming fast dynamics not only expressed in terms of matrices composed of blocks of regular stochastic matrices. Further, generalizations to non-autonomous and stochastic linear discrete systems are found in Sanz and Bravo de la Parra and Sanz

In Bravo de la Parra the aggregation of a class of nonlinear discrete system with two different time scales is developed. The one associated to the fast dynamics is used as time unit of the system, which is considered to be linear while the slow dynamics is supposed generally nonlinear. The system is transformed to make appear the global variables, and then a version of the center manifold theorem is applied to build up the aggregated system in a similar way to that used in thecase of ordinary differential equations.

In the construction of a discrete model with two time scales it is not always possible to choose as time unit the fast time unit. That is so because it may happen that during a fast time unit the action of the slow process is not describable. On the other hand, if the system is expressed in the slow time unit it is always possible to describe the action of the fast process during it by repeating a number of times its action during a fast unit. From this point of view it is even more interesting to extend the methods of aggregation for linear discrete systems expressed in the slow time unit to nonlinear cases. A first attempt to do it is found in Bravo de la Parra _. _. where the slow dynamics is linear and thus represented by a general nonnegative matrix, while the fast dynamics is dependent on global variables and is supposed to act a large number of times during one single time unit of the slow dynamics.

The aim of this work is to extend aggregation methods in the direction of the last mentioned work. We begin by proposing a very general discrete system including two different processes acting at different time scales. The time unit of the system is the one corresponding to the slow dynamics and the effect of the fast dynamics is represented assuming that the slow time unit is divided into a large number of fast time units and so that it acts a large number of times during one single slow time unit. Then, some 
assumptions, generalizing those required in previous works, are proposed which allow the construction of a reduced model associated to the original one. The following step consists of proving some results relating the solutions to the original and the reduced system. These results are not as general as those developed for systems of ordinary differential equations because we have not been able to transform the original system so that a version of the center manifold theorem applies. The summary of the main results is that it is possible to study the existence, stability and basins of attraction of steady states and periodic solutions to the original system performing this study for the reduced system.

The organization of the paper is as follows. In Section 2, we first present the reduction results obtained in previous works, then we propose a general nonlinear discrete time model of population dynamics with two time scales which, under some assumptions, can be reduced. Still in Section 2 we apply the presented reduction techniques in two particular examples. Section 3, which contains the main results of the paper, is devoted to the study of the relationship between the solutions to both systems, original and aggregated. For that, the reduction process is included in an abstract setting which contains as particular cases the models developed in Section 2. The advantage of this abstract approach lies in the fact that it simplifies the mathematical analysis and, moreover, it can be applied to the reduction of a more general class of nonlinear time discrete systems.

To make the reading easier, some technical proofs which are not necessary for the understanding of the text are deferred to a final Appendix.

\section{Discrete population models with two time scales}

In this section, we present a general population model with two time scales and summarize the reduction results obtained in previous works.

Let us suppose a population generally divided into groups, and each of these groups divided into several subgroups. We can think, for instance, of an age-structured population occupying a multi-patch environment. In this case, the population can be considered divided into groups which are the age classes, and each group divided into subgroups which are the individuals inhabiting each of the different patches.

The state at time $n$ of a population with $q$ groups is represented by a vector $X_{n}:=\left(x_{n}^{1}, \ldots, x_{n}^{q}\right)^{\top} \in \mathbb{R}_{+}^{N}$, where every vector $x_{n}^{i}:=\left(x_{n}^{i 1}, \ldots, x_{n}^{i N^{i}}\right) \in \mathbb{R}_{+}^{N^{i}}, i=1, \ldots, q$, represents the state of the $i$ group which is divided into $N^{i}$ subgroups, with $N=N^{1}+\cdots+N^{q}$.

In the evolution of the population we will consider two processes directly related to its structuring in groups and subgroups. We suppose that the characteristic time scales of both processes are very different from each other and so we will refer to them as the fast and the slow processes or, still, as the fast and the slow dynamics. If we start with the simplest case by considering both processes to be linear, we can represent them by two different matrices $F$ and $S$. The characteristic time scale of the fast process gives the projection interval associated to matrix $F$, that is, the state of the population, due to the fast process, after one fast time unit is $F X_{n}$. Analogously, the effect of the slow process after one slow time unit is calculated multiplying by matrix $S$. In order to write a single discrete model combining both processes, and therefore their different time scales, we have to choose its time unit. Two possible and reasonable choices are the time units associated to each one of the two processes. We use here as time unit of the model that corresponding to the slow dynamics, i.e. the time elapsed between times $n$ and $n+1$ is the projection interval associated to matrix $S$. We then need to approximate the effect of the fast dynamics over a time interval much longer than its own. In order to do so we will suppose that during each 
projection interval corresponding to the slow process matrix $F$ has operated a number $k$ of times, where $k$ is a big enough integer that can be interpreted as the ratio between the projection intervals corresponding to the slow and fast dynamics. Therefore, the fast dynamics will be modelled by $F^{k}$ and the proposed model will consist of the following system of $N$ linear difference equations that we will call general system:

$$
X_{k, n+1}=S F^{k} X_{k, n} .
$$

In order to reduce the system we must make some assumptions. Following Sánchez et al. [14] we suppose that for each group $i=1, \ldots, q$ the fast dynamics is internal, conservative of the total number of individuals and with an asymptotically stable (A.S.) distribution among the subgroups. These assumptions are met in the particular case of representing the fast dynamics for each group $i$ by a projection matrix $F_{i}$ which is a regular stochastic matrix of dimensions $N^{i} \times N^{i}$. The matrix $F$ that represents the fast dynamics for the whole population is then $F:=\operatorname{diag}\left(F_{1}, \ldots, F_{q}\right)$. Every matrix $F_{i}$ has an A.S. probability distribution $v_{i}$ which verifies that $F_{i} v_{i}=v_{i}$ and $u_{i} \cdot v_{i}=1$, where $u_{i}:=\left(1, \stackrel{\left(N^{i}\right)}{\stackrel{2}{P}}, 1\right)$.

The Perron-Frobenius theorem applies to matrix $F_{i}$ and we denote $\bar{F}_{i}:=\lim _{k \rightarrow \infty} F_{i}^{k}=\left(v_{i}|\ldots| v_{i}\right)=v_{i} u_{i}$, where $F_{i}^{k}$ is the $k$ th power of matrix $F_{i}$. Denoting $\bar{F}:=\operatorname{diag}\left(\bar{F}_{1}, \ldots, \bar{F}_{q}\right)$, we also have:

$$
\bar{F}=\lim _{k \rightarrow \infty} F^{k}=V U .
$$

where $V:=\operatorname{diag}\left(v_{1}, \ldots, v_{q}\right)_{N \times q}$ and $U:=\operatorname{diag}\left(u_{1}, \ldots, u_{q}\right)_{q \times N}$.

If we think that the ratio of slow to fast time scale tends to infinity, i.e. $k \rightarrow \infty$, or, in other words, that the fast process is instantaneous in relation to the slow process, we can approximate system (1) by the following so-called auxiliary system:

$$
X_{n+1}=S \bar{F} X_{n},
$$

which using (2) can be written as

$$
X_{n+1}=S V U X_{n} .
$$

Here we see that the evolution of the system depends on $U X_{n} \in \mathbb{R}^{q}$, which suggests that dynamics of the system could be described in terms of a lesser number of variables. These variables are usually called global variables, defined by

$$
Y_{n}:=U X_{n} .
$$

The auxiliary system (3) can be easily transformed into a $q$-dimensional system premultiplying by matrix $U$, giving rise to the so-called aggregated system:

$$
Y_{n+1}=U S V Y_{n} .
$$

The solutions to the auxiliary system can be obtained from the solutions to the aggregated system. It is straightforward that the solution $\left\{X_{n}\right\}_{n \in \mathbb{N}}$ of system (3) for the initial condition $X_{0}$ is related to the solution $\left\{Y_{n}\right\}_{n \in \mathbb{N}}$ of system (4) for the initial condition $Y_{0}=U X_{0}$ in the following way: $X_{n}=S V Y_{n-1}$ for every $n \geq 1$. The auxiliary system is an example of perfect aggregation in the sense of Iwasa 
Once the task of building up a reduced system is carried out, the important issue is to see if the dynamics of the general system (1) can also be studied by means of the aggregated system (4). For the simple linear case that we have just presented, it is proved in Sánchez that the asymptotic elements defining the long term behaviour of system (1) can be approximated by those of the corresponding aggregated system when the matrix associated to the latter is primitive. In Sanz and Bravo de la Parra these results are extended to more general linear cases. A generalization to a specific nonlinear case is treated in Bravo

\subsection{Reduction of a general non-linear model with two time scales}

Let us generalize the previous framework to include general nonlinear fast and slow processes.

First of all, let us present the so-called complete model which will be susceptible to being reduced. This model evolves in discrete time and is driven by two processes with different time scales: slow and fast. Such processes are defined respectively by two mappings

$$
S, F: \Omega_{N} \rightarrow \Omega_{N} ; S, F \in C^{1}\left(\Omega_{N}\right)
$$

where $\Omega_{N} \subset \mathbb{R}^{N}$ is a nonempty open set.

We choose as time step of the model that corresponding to the slow dynamics. In order to approximate the effect of the fast process over a time interval much bigger than its own, we assume that during this time step the fast process acts $k$ times before the slow process acts, where $k$ is a positive integer that in applications will take a big value.

Therefore, denoting by $X_{k, n} \in \mathbb{R}^{N}$ the vector of state variables at time $n$, the complete system is defined by

$$
X_{k, n+1}=S\left(F^{k}\left(X_{k, n}\right)\right)
$$

where $F^{k}$ denotes the $k$-fold composition of $F$ with itself.

In order to reduce the system (5), we have to impose some conditions on the fast process, which are specified in the following hypotheses.

Hypothesis 2.1. For each initial condition $X \in \Omega_{N}$, the fast dynamics tends to an equilibrium. That is, there exists a mapping

$$
\bar{F}: \Omega_{N} \rightarrow \Omega_{N}, \quad \bar{F} \in C^{1}\left(\Omega_{N}\right)
$$

such that

$$
\forall X \in \Omega_{N}, \quad \lim _{k \rightarrow \infty} F^{k}(X)=\bar{F}(X)
$$

Hypothesis 2.2. There exists a non-empty open set $\Omega_{q} \subset \mathbb{R}^{q}$ with $q<N$ and two mappings

$$
G: \Omega_{N} \rightarrow \Omega_{q}, \quad G \in C^{1}\left(\Omega_{N}\right) ; E: \Omega_{q} \rightarrow \Omega_{N}, E \in C^{1}\left(\Omega_{q}\right)
$$


such that $\bar{F}$ can be expressed as

$$
\bar{F}=E \circ G .
$$

In what follows we suppose that Hypotheses 2.1 and 2.2 are met. Then, we proceed to reduce system (5) in two steps. First, we define the so-called auxiliary system which approximates (5) when $k \rightarrow \infty$, i.e. when the fast process has reached an equilibrium. Denoting its vector of variables at time $n$ by $X_{n}$, this auxiliary system is

$$
X_{n+1}=S\left(\bar{F}\left(X_{n}\right)\right)=S \circ E \circ G\left(X_{n}\right) .
$$

Secondly, we define the global variables through

$$
Y_{n}:=G\left(X_{n}\right) \in \mathbb{R}^{q} .
$$

Applying $G$ to both sides of (6) we have

$$
Y_{n+1}=G\left(X_{n+1}\right)=G \circ S \circ E \circ G\left(X_{n}\right)=G \circ S \circ E\left(Y_{n}\right)
$$

which is an autonomous system in the global variables $Y_{n}$.

Summing up, we have approximated system (5) by the reduced or aggregated system defined by

$$
Y_{n+1}=G \circ S \circ E\left(Y_{n}\right) .
$$

Note that through the previous procedure we have constructed an approximation that allows us to reduce a system with $N$ variables to a new system with $q$ variables. In most practical applications, $q$ will be much smaller that $N$.

Coming back to Hypothesis 2.2, it can be interpreted by saying that for each initial condition $X \in \Omega_{N}$, the fast dynamics tends to an equilibrium which depends on $X$ only through $G(X)$, i.e. only through the value of the global variables corresponding to $X$.

\subsection{Two examples}

Before presenting the main results of this work in next section we illustrate their applicability by means of two examples. We first present a simple example where Proposition 3.10 applies. We consider a population living in an heterogeneous habitat, divided into $m$ patches. The migration process is assumed linear, described by a regular stochastic matrix $\mathcal{F}$ and fast in relation to demography. We denote $v=\left(v^{1}, \ldots v^{m}\right)$ the stable probability distribution of matrix $\mathcal{F}, u:=(1,(m), 1)$ and $\overline{\mathcal{F}}=\lim _{k \rightarrow \infty} \mathcal{F}^{k}=v^{\top} u$. Concerning demography we use on each patch the Beverton/Holt equation with different parameters to depict spatial heterogeneity. The state at time $n$ of the population is represented by vector $X_{n}:=\left(x_{n}^{1}, \ldots, x_{n}^{m}\right)^{\top} \in \mathbb{R}_{+}^{m}$. The model is

$$
X_{k, n+1}=S\left(\mathcal{F}^{k} X_{k, n}\right)
$$

where fast dynamics is defined by $F(X)=\mathcal{F} X$ and the slow dynamics by $S(X)=\left(b_{1} x^{1} /\left(1+c_{1} x^{1}\right), \ldots, b_{m} x^{m} /\left(1+c_{m} x^{m}\right)\right)$. The only global variable in this case is the total population $y=G(X)=\sum_{i=1}^{m} x^{i}$. We have $\bar{F}(X)=\overline{\mathcal{F}} X, E(y)=y v$ and, thus, the 
associated aggregated model is the following scalar difference equation

$$
y_{n+1}=f\left(y_{n}\right), \text { where } f(y)=G(S(E(y)))=\sum_{i=1}^{m} \frac{b_{i} v^{i}}{1+c_{i} v^{i} y} y
$$

This equation has an equilibrium 0 and the other equilibria are solutions to the equation

$$
1=g(y), \text { where } g(y)=\sum_{i=1}^{m} \frac{b_{i} v^{i}}{1+c_{i} v^{i} y}
$$

as $g(y)$ is decreasing for $y \in \mathbb{R}_{+}, g(0)=\sum_{i=1}^{m} b_{i} v^{i}$ and $\lim _{y \rightarrow \infty} g(y)=0$ there exists a positive equilibrium $y^{*}$ if and only if $\sum_{i=1}^{m} b_{i} v^{i}>1$. In this case, $y^{*}$ is the unique positive equilibrium. We proceed to study the asymptotic behaviour of solutions of the aggregated system with positive initial condition. We first observe that $f^{\prime}(y)=\sum_{i=1}^{m}\left(b_{i} v^{i} /(1+\right.$ $\left.\left.c_{i} v^{i} y\right)^{2}\right)>0$ and so $f(y)$ is an increasing function; moreover, we have $f^{\prime}(0)=\sum_{i=1}^{m} b_{i} v^{i}$ and $f^{\prime}\left(y^{*}\right)=\sum_{i=1}^{m}\left(b_{i} v^{i} /\left(1+c_{i} v^{i} y^{*}\right)^{2}\right)>0$. We distinguish two cases. In the first one we assume $f^{\prime}(0)=\sum_{i=1}^{m} b_{i} v^{i}<1$, then the only equilibrium is 0 which is hyperbolic and globally A.S. because $f(y)<y$ for $y>0$. In the second case we suppose $f^{\prime}(0)=\sum_{i=1}^{m} b_{i} v^{i}>1$, so the unique positive equilibrium $y^{*}$ is hyperbolic $\left(f^{\prime}\left(y^{*}\right)<\right.$ $\left.\sum_{i=1}^{m}\left(b_{i} v^{i} /\left(1+c_{i} v^{i} y^{*}\right)\right)=1\right)$ and globally A.S. because $f(y)>y$ when $y \in\left(0, y^{*}\right)$ and $f(y)<y$ when $y \in\left(y^{*}, \infty\right)$.

The results obtained for the aggregated model can be extended, applying Proposition 3.10 , to the original model as follows: For $k$ big enough, if $\sum_{i=1}^{m} b_{i} v^{i}<1$ the population gets extinct in the long term, whereas if $\sum_{i=1}^{m} b_{i} v^{i}>1$ it tends to an equilibrium $X_{k}^{*}$ which verifies $\lim _{k \rightarrow \infty} X_{k}^{*}=y^{*} v$.

In Proposition 3.10, the main results of this work are proved to apply to systems of the form

$$
X_{k, n+1}=\mathcal{S}\left(\mathcal{F}^{k} X_{k, n}\right)
$$

where $\mathcal{F}$ is a block-diagonal matrix with each block being a regular stochastic matrix as was supposed in the reduction of system (1). The previous example is a particular case of (8) with one single block in $\mathcal{F}$ and so, with a unique global variable.

Some examples of models described in terms of this kind of system are found in Charles and Lett They assume the fast process, migration, to be linear so that we can take $F(X)=\mathcal{F} X$. On the other hand, the slow process is defined by means of a density dependant matrix $\mathcal{L}(X)$ so that we can make $S(X)=\mathcal{L}(X) X$.

As a second example of the application of the results in next section we develop to a certain detail the model There is presented a general model of the dynamics of an age-structured (three age classes) Salmo trutta population inhabiting an arborescent river network divided into 15 patches at four levels. The aim of the model is the study of the influence of environmental managing (dams and channels) on the global population dynamics. The model couples both a linear discrete model for migrations and a non-linear density-dependent Leslie-type model for the demography, resulting in a system with $(15 \times 3)$ difference equations. The migration process is considered fast in relation to demography. The stability of the equilibria of the general model is studied carrying out the stability analysis of the corresponding equilibria of a reduced model with only three equations. 
To be precise, let $x_{n}^{i, j}$ be the number of individuals aged $i(i=1,2,3)$, on patch $j$ $(j=1, \ldots, 15)$ at time $n, x_{n}^{i}=\left(x_{n}^{i, 1}, \ldots, x_{n}^{i, 15}\right)$ and $X_{n}=\left(x_{n}^{1}, x_{n}^{2}, x_{n}^{3}\right) \in \mathbb{R}^{45}$. The migration process is considered to be linear and described by matrix $\mathcal{F}$, which is block-diagonal, $\mathcal{F}=\operatorname{diag}\left(F_{1}, F_{2}, F_{3}\right)$, where $F_{i}$ describes the movements between patches of the individuals of age-class $i$. Each $F_{i}$ is a regular stochastic matrix so that, following notations in (2), it possesses a probability distribution $v_{i}$ which verifies $\bar{F}_{i}:=\lim _{k \rightarrow \infty} F_{i}^{k}=$ $\left(v_{i}|\ldots| v_{i}\right)=v_{i} u_{i}$. It is also verified that $\overline{\mathcal{F}}=\lim _{k \rightarrow \infty} \mathcal{F}^{k}=V U$. The demography is also described by a matrix but, in this case, a non constant matrix; it depends on the total number of individuals in age class $1, y^{1}=u_{1} \cdot x^{1}$ :

$$
\mathcal{L}\left(y^{1}\right)=\left(\begin{array}{lll}
0 & 0 & f \\
s_{1}\left(y^{1}\right) & 0 & 0 \\
0 & s_{2} & s_{3}
\end{array}\right)
$$

where $s_{1}\left(y^{1}\right), s_{2}, s_{3}$ and $f$ are diagonal $15 \times 15$-blocks containing the demographical parameters (survival and fertility rates). The model including both processes at different time scales reads:

$$
X_{k, n+1}=\mathcal{L}\left(u_{1} \cdot\left(\mathcal{F}^{k} X_{k, n}\right)^{1}\right) \mathcal{F}^{k} X_{k, n}
$$

To place it in the framework of system 8 we have to take $F(X)=\mathcal{F} X$ and $S(X)=\mathcal{L}\left(u_{1} \cdot x^{1}\right) X$. So, $\bar{F}(X)=\overline{\mathcal{F}} X, \quad Y=G(X)=U X \in \mathbb{R}^{3} \quad$ and $E(Y)=V Y$. The associated aggregated system is

$$
Y_{n+1}=G \circ S \circ E\left(Y_{n}\right)=U \mathcal{L}\left(u_{1} \cdot\left(V Y_{n}\right)^{1}\right) V Y_{n}
$$

that is,

$$
Y_{n+1}=\left(\begin{array}{lcc}
0 & 0 & u_{1} f v_{3} \\
u_{2} s_{1}\left(y^{1}\right) v_{1} & 0 & 0 \\
0 & u_{3} s_{2} v_{2} & u_{3} s_{3} v_{3}
\end{array}\right) Y_{n}=\left(\begin{array}{lll}
0 & 0 & \bar{f} \\
\bar{s}_{1}\left(y^{1}\right) & 0 & 0 \\
0 & \bar{s}_{2} & \bar{s}_{3}
\end{array}\right) Y_{n}
$$

where, due to the particular definition of $s_{1}\left(y^{1}\right)$ we have $\bar{s}_{1}\left(y^{1}\right)=\alpha /\left(1+\beta y^{1}\right)$.

For system (10) it is easy to prove that, apart from the trivial equilibrium, if $\omega:=\alpha \bar{f} \bar{s}_{2}+\bar{s}_{2}-1>0$ there is a positive equilibrium

$$
Y^{*}=\left(y^{1 *}, y^{2 *}, y^{3 *}\right)=\left(\frac{\omega}{\beta\left(1-\bar{s}_{3}\right)}, \frac{\omega}{\beta \bar{f} \bar{s}_{2}}, \frac{\omega}{\beta \bar{f}\left(1-\bar{s}_{3}\right)}\right)
$$

which is hyperbolic A.S. whereas $(0,0,0)$ is hyperbolic unstable. When $\omega<0$ then $(0,0,0)$ is hyperbolic A.S.

These results can be extended to system (9) via Theorem 3.5 and Proposition 3.10. If $\omega>0$ then system (9), for big enough $k$, has a positive hyperbolic A.S. equilibrium $X_{k}^{*}$ well approximated by $X^{*}=\mathcal{L}\left(y^{1 *}\right) V Y^{*}$ and $\mathbf{0}=(0,45 ?, 0)$ is a hyperbolic unstable equilibrium. The basin of attraction of $X_{k}^{*}$ can be easily estimated starting from the basin of attraction of $Y^{*}$ in system (10). On the other hand, if $\omega<0$ then there is no positive equilibrium for system (9) and $\mathbf{0}$ is hyperbolic A.S. 


\section{A general formulation of the aggregation technique}

In this section, we will introduce a general non-linear model and an aggregation of variables procedure which contain as a particular case the setting developed in the previous section. The advantage of this new approach lies in the fact that it simplifies the mathematical calculations and, moreover, it could be applied to the reduction of more general systems as systems in which the existence of different time scales is associated to a real parameter in the model. With this aim, in our new formulation the parameter $k$ which differentiates the two time scales will be considered to be a big real number (not necessarily integer).

We start by introducing the original or complete model defined by

$$
X_{k, n+1}=H_{k}\left(X_{k, n}\right)
$$

where $k \in I \subset \mathbb{R}_{+}, I$ being a non-empty set not bounded from above and for each $k \in I$,

$$
H_{k}: \Omega_{N} \rightarrow \Omega_{N} ; H_{k} \in C^{1}\left(\Omega_{N}\right) .
$$

In order to carry out the reduction of the model, we assume the following conditions:

Hypothesis 3.1. The family of mappings $\left\{H_{k}\right\}_{k \in I}$ converges pointwise in $\Omega_{N}$ when $k \rightarrow \infty$ to a mapping $H: \Omega_{N} \rightarrow \Omega_{N}, H \in \mathrm{C}^{\mathrm{l}}\left(\Omega_{N}\right)$.

Hypothesis 3.2. There exist a non-empty open subset $\Omega_{q} \subset \mathbb{R}^{q}$ with $q<N$ and two mappings

$$
G: \Omega_{N} \rightarrow \Omega_{q} ; T: \Omega_{q} \rightarrow \Omega_{N} ; \quad G \in C^{1}\left(\Omega_{N}\right), \quad T \in C^{1}\left(\Omega_{q}\right)
$$

such that the mapping $H$ of Hypothesis 3.1 can be expressed as

$$
H=T \circ G .
$$

Let us observe that the setting of Section 2.1 corresponds to taking

$$
H_{k}:=S \circ F^{k} ; \quad H:=S \circ \bar{F}
$$

and Hypotheses 2.1 and 2.2 correspond respectively to Hypotheses 3.1 and 3.2 with $T:=S \circ E$

The approximate reduction of system (11) can be carried out as in Section 2.1. The auxiliary system is

$$
X_{n+1}=H\left(X_{n}\right)
$$

Defining the global variables by

$$
Y_{n}:=G\left(X_{n}\right) \in \mathbb{R}^{q}
$$

we obtain the reduced or aggregated system

$$
Y_{n+1}=\bar{H}\left(Y_{n}\right)
$$

where we have introduced the notation $\bar{H}:=G^{\circ} T$. 


\subsection{Relationship between the original system (11) and the aggregated system (13)}

In this section, we will relate the behaviour of systems (11) and (13), for big enough values of parameter $k$.

Our results are of two kinds: First we will compare the solutions of both systems for a fixed value of $n$. Secondly, we will give asymptotic results concerning the existence and stability of fixed points and periodic solutions for both systems. Moreover, we will show how the periodic orbits of the original system (11) can be approximated by those of the aggregated system (13).

Throughout this section we assume Hypotheses 3.1 and 3.2 and when comparing solutions we will always refer to the orbits corresponding to the initial condition $X_{0} \in \mathbb{R}^{N}$ for the original and the auxiliary systems and $Y_{0}:=G\left(X_{0}\right) \in \mathbb{R}^{q}$ for the aggregated system.

The following proposition guarantees that the dynamics of the auxiliary system (12) is completely determined by the dynamics of the reduced system (13), the converse holding too. Moreover, given mild extra assumptions, for each $n$ fixed we can approximate the solution to the original model (11) by the solution to the aggregated model (13).

Proposition 3.3. Let $X_{0} \in \Omega_{N}$ and let $Y_{0}:=G\left(X_{0}\right) \in \Omega_{q}$. Then:

(i) The solution $\left\{X_{n}\right\}_{n=1,2}$ to the auxiliary system (12) corresponding to the initial condition $X_{0}$ and the solution $\left\{Y_{n}\right\}_{n=1,2, \ldots}$ to the reduced system (13) corresponding to the initial condition $Y_{0}$ are related by the following expressions

$$
Y_{n}=G\left(X_{n}\right) ; \quad X_{n}=T\left(Y_{n-1}\right), \quad n=1,2, \ldots
$$

(ii) Let $n$ be a fixed positive integer and let us assume that there exists a non-empty bounded and open set $\Omega$ such that $\bar{\Omega} \subset \Omega_{N}, \Omega$ contains the points $X_{0}, X_{i+1}:=T\left(Y_{i}\right)$, $i=0, \ldots, n-1$ and such that $\lim _{k \rightarrow \infty} H_{k}=H$ uniformly in $\Omega$. Then the solution $X_{k, n}$ to the original system (11) corresponding to the initial condition $X_{0}$ and the solution $Y_{n}$ to the reduced system (13) corresponding to the initial condition $Y_{0}$ are related by the following expressions

$$
Y_{n}=\lim _{k \rightarrow \infty} G\left(X_{k, n}\right) ; \quad \lim _{k \rightarrow \infty} X_{k, n}=T\left(Y_{n-1}\right) .
$$

Proof.

(i) The first expression holds by the definition of the global variables. Regarding the second one, we have

$$
X_{n}=H\left(X_{n-1}\right)=T \circ G\left(X_{n-1}\right)=T\left(Y_{n-1}\right)
$$

as we wanted to show.

(ii) Bearing in mind (14), we only need to show that $\lim _{k \rightarrow \infty} X_{k, n}=X_{n}$, i.e. that $\lim _{k \rightarrow \infty} H_{k}^{n}\left(X_{0}\right)=H^{n}\left(X_{0}\right)$.

This equality is an immediate consequence of a technical result which is stated as Lemma A.1 in the Appendix.

Now we will turn our attention to the study of some relationships between the fixed points and periodic solutions to the original and reduced systems.

Let us recall that $X^{*}$ is a periodic point of period $m$ or an m-periodic point for system $X_{n+1}=A\left(X_{n}\right)$ if $X^{*}=A^{m}\left(X^{*}\right)$ and $m$ is the lowest positive integer for which this equality 
holds. In the case $m=1, X^{*}$ is called an equilibrium point or fixed point. An $m$-periodic point $X^{*}$ is said to be hyperbolic if none of the eigenvalues of $D A^{m}\left(X^{*}\right)$, differential of $A^{m}$ at $X^{*}$, has modulus 1 . If all the eigenvalues of $D A^{m}\left(X^{*}\right)$ have modulus less than 1 then $X^{*}$ is A.S. and the set of initial conditions which corresponding solutions tend to it is called the basin of attraction. If any of the eigenvalues of $D A^{m}\left(X^{*}\right)$ have modulus larger than 1 then $X^{*}$ is unstable

First of all we relate the existence of fixed and periodic points and their stability for the auxiliary system (12) and the aggregated system (13).

\section{Proposition 3.4.}

(a) If $X^{*} \in \Omega_{N}$ is a periodic point of period $m \geq 1$ of the auxiliary system (12), then $Y^{*}:=G\left(X^{*}\right) \in \Omega_{q}$ is a periodic point of period $m$ of the reduced system (13). Conversely, if $Y^{*} \in \Omega_{q}$ is a periodic point of period $m \geq 1$ of the reduced system (13) then $X^{*}:=T\left(Y^{*}\right) \in \Omega_{N}$ is a periodic point of period $m$ of the auxiliary system (12).

(b) Let $Y^{*} \in \Omega_{q}$ be an m-periodic hyperbolic point of (13) and $X^{*}:=S\left(Y^{*}\right)$. Then $X^{*}$ is (A.S.) (resp. unstable) for (12) if and only if $Y^{*}$ is A.S. (resp. unstable) for (13). Conversely, if $X^{*} \in \Omega_{N}$ is an m-periodic hyperbolic point of $(12)$ and $Y^{*}:=G\left(X^{*}\right)$, then $Y^{*}$ is A.S. (resp. unstable) for (13) if and only if $X^{*}$ is A.S. (resp. unstable) for (12).

Proof. See appendix.

The following result, which is the main result of this paper, relates the periodic points for the original system (11) and the aggregated system (13). Roughly speaking, it guarantees that under certain assumptions, the existence of an $m$-periodic point $Y^{*}$ for the aggregated system implies, for big enough values of $k$, the existence of an $m$-periodic point $X_{k}^{*}$ for the original system, which can be approximated in terms of $Y^{*}$. Moreover, in the hyperbolic case the stability of $Y^{*}$ is equivalent to the stability of $X_{k}^{*}$ and in the A.S. case, the basin of attraction of $X_{k}^{*}$ can be approximated in terms of the basin of attraction of $Y^{*}$.

THEOREM 3.5. Let $Y^{*} \in \Omega_{q}$ be an m-periodic point of the aggregated model (13) so that, according to Proposition 3.4, $X^{*}:=T\left(Y^{*}\right)$ is an m-periodic point for the auxiliary system (12).

Let us assume the following hypothesis:

Hypothesis 3.6. There exists a non-empty bounded open set $\Omega$ such that $\bar{\Omega} \subset \Omega_{N}$, which contains the set

$$
\mathcal{H}_{m-1}\left(X^{*}\right):=\left\{X^{*}, H\left(X^{*}\right), \ldots, H^{m-1}\left(X^{*}\right)\right\}
$$

and such that

$$
\lim _{k \rightarrow \infty} H_{k}=H ; \quad \lim _{k \rightarrow \infty} D H_{k}=D H
$$

uniformly in $\Omega$.

In addition to Hypothesis 3.6, let us assume that 1 is not an eigenvalue of $D \bar{H}^{m}\left(Y^{*}\right)$. Then there exist $r_{0}>0, k_{0} \in I$ such that: 
(a) For each $k \geq k_{0}, k \in I, H_{k}$ has an unique $m$-periodic point $X_{k}^{*} \in \bar{B}\left(X^{*} ; r_{0}\right)$.

(b) $\lim _{k \rightarrow \infty} X_{k}^{*}=X^{*}$.

(c) Let $Y^{*}$ be hyperbolic. Then there exists $k_{0}^{*}$ such that for all $k \geq k_{0}^{*}, k \in I, X_{k}^{*}$ is hyperbolic. Moreover, $X_{k}^{*}$ is A.S. (resp. unstable) if and only if $Y^{*}$ is A.S. (resp. unstable).

(d) Let $Y^{*}$ be hyperbolic and A.S. Let $X_{0} \in \Omega_{N}$ such that $Y_{0}:=G\left(X_{0}\right)$ verifies $\lim _{n \rightarrow \infty} \bar{H}^{m n-1}\left(Y_{0}\right)=Y^{*}$. Let us assume the following hypothesis:

Hypothesis 3.7. There exists an open set $Q \subset \Omega_{N}$ containing the set

$$
\mathcal{H}\left(X_{0}\right):=\left\{X_{0}, X_{n+1}:=T\left(Y_{n}\right), \quad n=0,1, \ldots\right\}
$$

such that $\lim _{k \rightarrow \infty} H_{k}=H$ uniformly in $Q$.

Then there exists $k_{0}^{* *}$ such that for each $k \geq k_{0}^{*}, k \in I, \lim _{n \rightarrow \infty} H_{k}^{m n}\left(X_{0}\right)=X_{k}^{*}$.

As usual, $\bar{B}(X ; r)$ represents the set $\left\{Z \in \mathbf{R}^{N} ;\|X-Z\| \leq r\right\}$.

Proof. See Appendix.

COROLlaRY 3.8. Let us assume that $H_{k}, H \in C^{1}\left(\Omega_{N}\right)$ and that $\lim _{k \rightarrow \infty} H_{k}=H$, $\lim _{k \rightarrow \infty} D H_{k}=D H$ uniformly on any compact set $K \subset \Omega_{N}$.

Let $Y^{*} \in \mathbb{R}^{q}$ be a hyperbolic and A.S. equilibrium point of (13). Then there exists $k_{0} \in I$ such that for each $k \geq k_{0}, k \in I$, there exists an equilibrium point $X_{k}^{*}$ of (11) which is hyperbolic and A.S. and that satisfies $\lim _{k \rightarrow \infty} X_{k}^{*}=X^{*}$ where $X^{*}=T\left(Y^{*}\right)$.

Moreover, if $X_{0} \in \mathbb{R}^{N}$ is such that $\lim _{n \rightarrow \infty} \bar{H}^{n}\left(Y_{0}\right)=Y^{*}$, where $Y_{0}:=G\left(X_{0}\right)$, then

$$
\forall k \geq k_{0}, \quad k \in I, \quad \lim _{n \rightarrow \infty} H_{k}^{n}\left(X_{0}\right)=X_{k}^{*}
$$

The applicability of Theorem 3.5 depends on Hypotheses 3.6 and 3.7, which require the uniform convergence of the mappings $H_{k}$ and $D H_{k}$ to $H$ and $D H$, respectively, in a certain set. Coming back to the context of a system with two time scales of Section 2.1, the following proposition establishes sufficient conditions on the fast process $F$ under which we can guarantee these hypotheses hold. The proof is standard and is omitted.

Proposition 3.9. Under the conditions of Section 2.1, let $\Omega$ be any non-empty open bounded set such that $\bar{\Omega} \subset \Omega_{N}$ and let us assume that:

(i) $\lim _{k \rightarrow \infty} F^{k}=\bar{F}$ uniformly in $\bar{\Omega}$.

(ii) $\lim _{k \rightarrow \infty} D F^{k}=D \bar{F}$ uniformly in $\bar{\Omega}$.

Then mappings $H_{k}:=S \circ F^{k}$ and $H:=S \circ \bar{F}$ verify:

(a) $\lim _{k \rightarrow \infty} H_{k}=H$ uniformly in $\Omega$.

(b) $\lim _{k \rightarrow \infty} D H_{k}=D H$ uniformly in $\Omega$.

We now state in the following proposition sufficient conditions for system 8 , $X_{k, n+1}=S\left(\mathcal{F}^{k} X_{k, n}\right)$, to meet the hypotheses of Theorem 3.5. 
Proposition 3.10. Let us consider system (8) as particular case of system (5) with $F(X)=\mathcal{F} X$ where matrix $\mathcal{F}$ is a block-diagonal matrix with each block being a regular stochastic matrix as in (1), $\overline{\mathcal{F}}=\lim _{k \rightarrow \infty} \mathcal{F}^{k}=V U$ and with $S$ a $C^{1}$ map from $\Omega_{N}$ to $\mathbb{R}^{m}$. Then Hypotheses 2.1 and 2.2 are met with $G(X)=U X$ and $E(Y)=V Y$ and the corresponding aggregated system is $Y_{n+1}=U S\left(V Y_{n}\right)$. Moreover, if $Y^{*}$ is an m-periodic point of the aggregated system then Hypotheses 3.6 and 3.7 are met and Theorem 3.5 applies to system (8).

Proof. Let $\|\cdot\|$ be any consistent matrix norm and let $\Omega \subset R_{N}$ be bounded. Then

$$
\sup _{X \in \bar{\Omega}}\left\|F^{k}(X)-\bar{F}(X)\right\|=\sup _{X \in \bar{\Omega}}\left\|\mathcal{F}^{k} X-\overline{\mathcal{F}} X\right\| \leq\left\|\mathcal{F}^{k}-\overline{\mathcal{F}}\right\| \sup _{X \in \bar{\Omega}}\|X\| \underset{k \rightarrow \infty}{\rightarrow} 0
$$

Similarly $\sup _{X \in \bar{\Omega}}\left\|D F^{k}(X)-D \bar{F}(X)\right\|=\left\|\mathcal{F}^{k}-\overline{\mathcal{F}}\right\|_{k \rightarrow \infty}^{\rightarrow 0} 0$ and so the uniform convergence of $F^{k}$ and $D F^{k}$ to $\bar{F}$ and $D \bar{F}$ in $\bar{\Omega}$ holds and Proposition 3.9 applies.

\section{Conclusion}

In the present work we have introduced, Section 2, a very general discrete nonlinear model where we have distinguished between two different time scales. The model is represented by a discrete system at the slow time scale and, with the help of suitable assumptions, is reduced to a simpler system, also at the slow time scale, which terms reflect the asymptotic behaviour of the fast dynamics. This is an example of how a simpler model admits an explanation given by a more complex model. The study of the simpler model, the aggregated model, give us information of the initial model via the general results of Section 3.

The results of Section 3 need some hypotheses not necessarily met for the general systems presented in Section 2. To illustrate the importance of Theorem 3.5 we mention at the end of Section 2 two applications which can be included in a general kind of two-time scales discrete systems where the fast dynamics is linear. For this kind of systems Proposition 3.10 assures that hypotheses of Theorem 3.5 hold, which justifies the use of aggregation methods in the study of these systems. In both applications the influence of spatial heterogeneity on the stability of ecological communities is studied. Several new applications could also be developed in the same direction in the future. The aggregation methods are particularly suited for the study of spatially distributed populations in a network of patches connected by migrations because they allow a simpler system of equations governing the dynamics of the total populations to be obtained.

From a more methodological point of view, there are two issues to develop: the first one is to characterize larger classes of discrete systems where the aggregation results can be easily applied, that is, generalizing Proposition 3.10 and the second one has to do with the possibility of extending Theorem 3.5 to more general asymptotic behaviours.

\section{Acknowledgements}

The authors are supported by Ministerio de Educación y Ciencia (Spain), proyecto MTM2005-00423 and FEDER. 


\section{References}

P. Auger, Dynamics and Thermodynamics in Hierarchically Organized Systems. Pergamon Press, Oxford, 1989.

P. Auger and R. Roussarie, Complex ecological models with simple dynamics: From individuals to populations, Acta Biotheor. 42 (1994), pp. 111-136.

R. Bravo de la Parra, P. Auger, and E. Sánchez, Aggregation methods in discrete models, J. Biol. Syst. 3 (1995), pp. 603-612.

R. Bravo de la Parra et al., A discrete model with density dependent fast migration, Math. Biosci. 157 (1999), pp. $91-110$.

R. Bravo de la Parra, E. Sánchez, and P. Auger, Time scales in density dependent discrete models, J. Biol. Syst. 5 (1997), pp. 111-129.

S. Charles et al., Population dynamics modelling in an hierarchical arborescent river network: An attempt with Salmo trutta, Acta Biotheor. 46 (1998), pp. 223-234.

$\mathrm{S}$. Charles et al., A density dependent model describing Salmo trutta population dynamics in an arborescent river network: Effects of dams and channelling, C. R. Acad. Sci. Paris, Sciences de la vie 321 (1998), pp. $979-990$.

N. Fenichel, Persistence and smoothness of invariant manifolds for flows, Ind. Univ. Math. J. 21 (1971), pp. $193-226$.

R.A. Horn and C.A. Johnson, Matrix Analysis, Cambridge University Press, Cambridge, 1985.

Y. Iwasa, V. Andreasen, and S.A. Levin, Aggregation in model ecosystems I. Perfect aggregation, Ecol. Modelling 37 (1987), pp. 287-302.

C. Lett, P. Auger, and R. Bravo de la Parra, Migration frequency and the persistence of host-parasitoid interactions, J. Theor. Biol. 221 (2003), pp. 639-654.

C. Lett, P. Auger, and F. Fleury, Effects of asymmetric dispersal and environmental gradients on the stability of host-parasitoid systems, Oikos 109 (2005), pp. 603-613.

R.C. Robinson, Dynamical Systems: Stability, Symbolic Dynamics and Chaos, CRC Press, London, 1995. E. Sánchez, R. Bravo de la Parra, and P. Auger, Discrete models with different time-scales, Acta Biotheor. 43 (1995), pp. $465-479$.

L. Sanz, A. Blasco, and R. Bravo de la Parra, Approximate reduction of multi-type Galton-Watson processes with two time scales, Math. Models Methods Appl. Sci. 13 (2003), pp. 491-525.

L. Sanz and R. Bravo de la Parra, Variables aggregation in a time discrete linear model, Math. Biosci. 157 (1999), pp. $111-146$.

L. Sanz and R. Bravo de la Parra, Time scales in stochastic multiregional models, Nonlinear Anal. Real World Appl. 1 (2000), pp. 89-122.

L. Sanz and R. Bravo de la Parra, Time scales in a non autonomous linear discrete model, Math. Models Methods Appl. Sci. 11 (2001), pp. 1203-1235.

G.W. Stewart and J.I. Guang Sun, Matrix Perturbation Theory, Academic Press, Boston, 1990.

\section{Appendix A Proofs}

In what follows we will denote by $\|\cdot\|$, depending on the context, a norm in $\mathbb{R}^{p}$ or its associated matrix norm in $\mathbb{R}^{p \times p}$, with $p=N, q$.

\section{Proof of Proposition 3.4.}

(a) For each $m \geq 1$ the following holds:

$$
\begin{aligned}
& H^{m}=(T \circ G)^{m}=T \circ \bar{H}^{m-1} \circ G \\
& \bar{H}^{m}=(G \circ T)^{m}=G \circ H^{m-1} \circ T
\end{aligned}
$$

Let $X^{*}$ be an $m$-periodic point of $(12)$, so $H^{m}\left(X^{*}\right)=X^{*}$. Using (A1) we have

$$
Y^{*}=G\left(X^{*}\right)=G\left(H^{m}\left(X^{*}\right)\right)=G \circ T \circ \bar{H}^{m-1} \circ G\left(X^{*}\right)=\bar{H}^{m}\left(G\left(X^{*}\right)\right)=\bar{H}^{m}\left(Y^{*}\right) .
$$

In order to show that $m$ is the lowest integer for which (A3) holds, let us assume that there exists $s<m$ such that $\bar{H}^{s}\left(Y^{*}\right)=Y^{*}$, i.e.

$$
G\left(X^{*}\right)=G \circ H^{s-1} \circ T\left(G\left(X^{*}\right)\right)=G^{\circ} H^{s}\left(X^{*}\right) .
$$


Applying $T$ to both sides of the equality above we have

$$
T \circ G\left(X^{*}\right)=H\left(X^{*}\right)=T \circ G \circ H^{s}\left(X^{*}\right)=H^{s+1}\left(X^{*}\right)
$$

and then

$$
X^{*}=H^{m}\left(X^{*}\right)=H^{m-s-1} \circ H^{s+1}\left(X^{*}\right)=H^{m-s-1} \circ H\left(X^{*}\right)=H^{m-s}\left(X^{*}\right)
$$

which contradicts the assumption of $X^{*}$ being $m$-periodic.

Conversely, let us assume that $\bar{H}^{m}\left(Y^{*}\right)=Y^{*}$. Then using (A2) we have for $X^{*}:=T\left(Y^{*}\right)$ :

$$
H^{m}\left(X^{*}\right)=T \circ \bar{H}^{m-1} \circ G\left(T\left(Y^{*}\right)\right)=T \circ \bar{H}^{m}\left(Y^{*}\right)=T\left(Y^{*}\right)=X^{*} .
$$

In order to show that $m$ is the lowest integer for which (A4) holds, let us assume that there exists $s<m$ such that $H^{s}\left(X^{*}\right)=X^{*}$. Then, it follows from (A2) that

$$
\bar{H}^{s}\left(Y^{*}\right)=G^{\circ} H^{s-1} \circ T\left(Y^{*}\right)=G^{\circ} H^{s-1} \circ T\left(G\left(X^{*}\right)\right)=G^{\circ} H^{s}\left(X^{*}\right)=G\left(X^{*}\right)=Y^{*}
$$

which contradicts the assumption of $Y^{*}$ being $m$-periodic.

(b) Let $X^{*}$ be such that $H^{m}\left(X^{*}\right)=X^{*}$ and let $Y^{*}:=G\left(X^{*}\right)$. Then we have

$$
D H^{m}\left(X^{*}\right)=D\left(H^{m-1} \circ T \circ G\right)\left(X^{*}\right)=D\left(H^{m-1} \circ T\right)\left(Y^{*}\right) D G\left(X^{*}\right)
$$

and also

$$
D \bar{H}^{m}\left(Y^{*}\right)=D\left(G \circ H^{m-1} \circ T\right)\left(Y^{*}\right)=D G\left(X^{*}\right) D\left(H^{m-1} \circ T\right)\left(Y^{*}\right)
$$

where we have used that

$$
H^{m-1} \circ T\left(Y^{*}\right)=H^{m-1} \circ T\left(G\left(X^{*}\right)\right)=H^{m}\left(X^{*}\right)=X^{*} .
$$

Conversely, let $Y^{*}$ be such that $\bar{H}^{m}\left(Y^{*}\right)=Y^{*}$ and let $X^{*}:=T\left(Y^{*}\right)$. Similar calculations to the ones before show that

$$
D \bar{H}^{m}\left(Y^{*}\right)=D\left(\bar{H}^{m-1} \circ G\right)\left(X^{*}\right) D T\left(Y^{*}\right)
$$

and

$$
D H^{m}\left(X^{*}\right)=D T\left(Y^{*}\right) D\left(\bar{H}^{m-1} \circ G\right)\left(X^{*}\right) .
$$

Summing up, we conclude that in both cases, $D H^{m}\left(X^{*}\right)$ and $D \bar{H}^{m}\left(Y^{*}\right)$ can be written as the product of two factors which are the same but in a different order. Therefore, both matrices must have the same non-zero eigenvalues ([9]).

More specifically,

$$
\sigma\left(D H^{m}\left(X^{*}\right)\right)=\sigma\left(D \bar{H}^{m}\left(Y^{*}\right)\right) \cup\left\{0,{ }^{(N-q)}, 0\right\}
$$

and so in particular,

$$
\rho\left(D H^{m}\left(X^{*}\right)\right)=\rho\left(D \bar{H}^{m}\left(Y^{*}\right)\right)
$$

(as usual, $\sigma(A)$ denotes the set of eigenvalues of matrix $A$, including multiplicities).

Henceforth, $\rho\left(D H^{m}\left(X^{*}\right)\right)<1$ (resp. $>1$ ) if and only if $\rho\left(D \bar{H}^{m}\left(Y^{*}\right)\right)<1$ (resp. $>1$ ) which concludes the proof of (b).

Now we establish two technical lemmas which will be useful in proving Theorem 3.5. 
LeMma A.1. Let $X_{0} \in \Omega_{N}$ and $n \in \mathbf{Z}_{+}$be fixed. Let $\Omega$ be a non-empty open set such that $\bar{\Omega} \subset \Omega_{N}$ and which contains the set

$$
\mathcal{H}_{n}\left(X_{0}\right):=\left\{X_{0}, H\left(X_{0}\right), \ldots, H^{n}\left(X_{0}\right)\right\}
$$

Let us assume that $\lim _{k \rightarrow \infty} H_{k}=H$ uniformly to in $\Omega$. Then, there exists $r^{*}>0$ such that for each $s=1, \ldots, n$ we have $\lim _{k \rightarrow \infty} H_{k}^{s}=H^{s}$ uniformly in $\bar{B}\left(X_{0} ; r^{*}\right)$. If in addition $\lim _{k \rightarrow \infty} D H_{k}=D H$ uniformly in $\Omega$, then for each $s=1, \ldots, n$ we have $\lim _{k \rightarrow \infty} D H_{k}^{s}=$ $D H^{s}$ uniformly in $\bar{B}\left(X_{0} ; r^{*}\right)$.

Proof. It is easy to realize that it suffices with proving the result for $n=2$.

Since $X_{0}, H\left(X_{0}\right) \in \Omega$ and $\Omega$ is an open set, there exists $r>0$ such that $\bar{B}\left(X_{0} ; r\right) \subset \Omega$ and $\bar{B}\left(H\left(X_{0}\right) ; r\right) \subset \Omega$.

Moreover, since $\lim _{k \rightarrow \infty} H_{k}=H$ uniformly in $\Omega$ and $H$ is continuous in $X_{0}$, straightforward calculations show that there exist $r^{*}>0$ and $k^{*} \in I$ such that $\forall k \geq k^{*}, k \in I$, we have

$$
X \in \bar{B}\left(X_{0} ; r^{*}\right) \Rightarrow H(X), H_{k}(X) \in \bar{B}\left(H\left(X_{0}\right) ; r\right)
$$

Let us now show that $\lim _{k \rightarrow \infty} H_{k}^{2}=H^{2}$ uniformly in $\bar{B}\left(X_{0} ; r^{*}\right)$.

The uniform convergence $\lim _{k \rightarrow+\infty} H_{k}=H$ in $\Omega$ assures the existence of a real sequence $\left\{\alpha_{k}\right\}_{k \in I}, \alpha_{k}>0$, with $\lim _{k \rightarrow \infty} \alpha_{k}=0$ and such that

$$
\sup _{X \in \Omega}\left\|H_{k}(X)-H(X)\right\| \leq \alpha_{k}
$$

Since,

$$
\left\|H_{k}^{2}(X)-H^{2}(X)\right\|=\left\|H_{k}\left(H_{k}(X)\right)-H\left(H_{k}(X)\right)\right\|+\left\|H\left(H_{k}(X)\right)-H(H(X))\right\|
$$

we have, for $k \geq k^{*}$

$$
\sup _{X \in \bar{B}\left(X_{0} ; r^{*}\right)}\left\|H_{k}^{2}(X)-H^{2}(X)\right\| \leq \sup _{Z \in \bar{B}\left(H\left(X_{0}\right) ; r\right)}\left\|H_{k}(Z)-H(Z)\right\|+\sup _{\substack{Z_{k}, Z \in \bar{B}\left(H\left(X_{0}\right) ; r\right) \\ \| Z_{k}} \geq \| \leqslant \alpha_{k}}\left\|H\left(Z_{k}\right)-H(Z)\right\| .
$$

When $k \rightarrow \infty$, the first term on the right-hand side converges to zero due to the uniform convergence of $H_{k}$ to $H$ in $\Omega$ and the second term converges to zero since $H$ is uniformly continuous in $\bar{\Omega}$.

Now let us prove that $\lim _{k \rightarrow \infty} D H_{k}^{2}=D H^{2}$ uniformly in $\bar{B}\left(X_{0} ; r^{*}\right)$, under the additional assumption that $\lim _{k \rightarrow \infty} D H_{k}=D H$ uniformly in $\Omega$.

Since,

$$
\begin{aligned}
\left\|D H_{k}^{2}(X)-D H^{2}(X)\right\|= & \left\|D H_{k}\left(H_{k}(X)\right) D H_{k}(X)-D H(H(X)) D H(X)\right\| \\
\leq & \left\|D H_{k}\left(H_{k}(X)\right) D H_{k}(X)-D H\left(H_{k}(X)\right) D H_{k}(X)\right\| \\
& +\left\|D H\left(H_{k}(X)\right) D H_{k}(X)-D H(H(X)) D H_{k}(X)\right\| \\
& +\left\|D H(H(X)) D H_{k}(X)-D H(H(X)) D H(X)\right\|
\end{aligned}
$$


we have, for $k>k *$

$$
\begin{aligned}
(\mathrm{I}) & :=\sup _{X \in \bar{B}\left(X_{0} ; r^{*}\right)}\left\|D H_{k}\left(H_{k}(X)\right) D H_{k}(X)-D H\left(H_{k}(X)\right) D H_{k}(X)\right\| \\
& \leq \sup _{X \in \bar{B}\left(X_{0} ; r^{*}\right)}\left\|D H_{k}\left(H_{k}(X)\right)-D H\left(H_{k}(X)\right)\right\| \sup _{X \in \bar{B}\left(X_{0} ; r^{*}\right)}\left\|D H_{k}(X)\right\| \\
& \leq \sup _{Z \in \bar{B}\left(H\left(X_{0}\right) ; r\right)}\left\|D H_{k}(Z)-D H(Z)\right\| \sup _{X \in \bar{B}\left(X_{0} ; r^{*}\right)}\left\|D H_{k}(X)\right\| \underset{k \rightarrow \infty}{\rightarrow} 0
\end{aligned}
$$

where we have used the uniform convergence of $D H_{k}$ to $D H$ in $\Omega$ and the fact that, since $D H_{k} \rightarrow D H$ uniformly in $\Omega$ and $D H$ is continuous in $\bar{\Omega}$, then $\left\|D H_{k}(X)\right\|$ is uniformly bounded with respect to $k$ in $\bar{\Omega}$.

Similarly

$$
\begin{aligned}
\text { (II) } & :=\sup _{X \in \bar{B}\left(X_{0} ; r^{*}\right)}\left\|D H\left(H_{k}(X)\right) D H_{k}(X)-D H(H(X)) D H_{k}(X)\right\| \\
& \leq \sup _{X \in \bar{B}\left(X_{0} ; r^{*}\right)}\left\|D H\left(H_{k}(X)\right)-D H(H(X))\right\| \sup _{X \in \bar{B}\left(X_{0} ; r^{*}\right)}\left\|D H_{k}(X)\right\| \\
& \leq \sup _{\substack{Z_{k}, Z \in \bar{B}\left(H\left(X_{0}\right) ; r\right) \\
\left\|Z_{k} Z\right\| \leq_{k}}}\left\|D H\left(Z_{k}\right)-D H(Z)\right\| \sup _{X \in \bar{B}\left(X_{0} ; r^{*}\right)}\left\|D H_{k}(X)\right\| \rightarrow 0 .
\end{aligned}
$$

Finally, we have

$$
\begin{aligned}
(\mathrm{III}) & :=\sup _{X \in \bar{B}\left(X_{0} ; r^{*}\right)}\left\|D H(H(X)) D H_{k}(X)-D H(H(X)) D H(X)\right\| \\
& \left.\leq \sup _{X \in \bar{B}\left(X_{0} ; r^{*}\right)}\|D H(H(X))\| \sup _{X \in \bar{B}\left(X_{0} ; r^{*}\right)} \| D H_{k}(X)-D H(X)\right) \| \\
& \left.\leq \sup _{Z \in \bar{B}\left(H\left(X_{0}\right) ; r\right)}\|D H(Z)\| \sup _{X \in \bar{B}\left(X_{0} ; r^{*}\right)} \| D H_{k}(X)-D H(X)\right) \| \rightarrow 0 .
\end{aligned}
$$

Therefore the result is proved.

LeMmA A.2. Let Hypothesis 3.6 holds and assume that 1 is not an eigenvalue of $D H^{m}\left(X^{*}\right)$. Then there exist $\hat{r}>0, \hat{k} \in I$ such that for all $k \geq \hat{k}, k \in I$, the mappings

$$
W(X):=X-H^{m}(X) ; \quad W_{k}(X):=X-H_{k}^{m}(X)
$$

are one-to-one in $\bar{B}\left(X^{*} ; \hat{r}\right) \subset \Omega$.

Proof. First of all, let us recall the fact that if a sequence of matrices $B_{k}$ converges to a regular matrix $B$, then matrices $B_{k}$ are regular for $k$ big enough and moreover $\lim _{k \rightarrow \infty} B_{k}^{-1}=B^{-1}$ ([19]).

Differentiating in (A5) we have $D W\left(X^{*}\right)=I-D H^{m}\left(X^{*}\right)$ and since 1 is not an eigenvalue of $D H^{m}\left(X^{*}\right)$, it follows that $D W\left(X^{*}\right)$ is a regular matrix.

Hypothesis 3.6 allows us to apply Lemma A.1 and so there exists $r^{*}>0$ such that $\lim _{k \rightarrow \infty} D H_{k}^{m}=D H^{m}$ uniformly in $\bar{B}\left(X^{*} ; r^{*}\right)$, and consequently $\lim _{k \rightarrow \infty} D W_{k}=D W$ uniformly in $\bar{B}\left(X^{*} ; r^{*}\right)$. Therefore there exists $k_{1} \in I$ such that for all $k \geq k_{1}, k \in I, D W_{k}\left(X^{*}\right)$ is a regular matrix and moreover

$$
\lim _{k \rightarrow \infty}\left[D W_{k}\left(X^{*}\right)\right]^{-1}=\left[D W\left(X^{*}\right)\right]^{-1}
$$


In particular, this implies that there exists $k_{2} \in I$ such that $\forall k \geq k_{2}, k \in I$ we have

$$
\left\|A_{k}\right\| \leq 2\|A\|
$$

where we have denoted $A_{k}:=\left[D W_{k}\left(X^{*}\right)\right]^{-1}$ and $A:=\left[D W\left(X^{*}\right)\right]^{-1}$.

Since $D W$ is continuous in $X^{*}$, there exists $\eta>0$ such that

$$
X \in \bar{B}\left(X^{*} ; \eta\right) \Rightarrow\left\|D W(X)-D W\left(X^{*}\right)\right\| \leq \frac{1}{3\|A\|} .
$$

Also, since $\lim _{k \rightarrow \infty} D W_{k}=D W$ uniformly in $\bar{B}\left(X^{*} ; r^{*}\right)$, there exists $k_{3} \in I$ such that $\forall k \geq k_{3}$, $k \in I$

$$
\sup _{X \in \bar{B}\left(X^{*} ; r^{*}\right)}\left\|D W_{k}(X)-D W(X)\right\| \leq \frac{1}{24\|A\|} .
$$

We want to prove that for each $Z \in \mathbb{R}^{N}$, both the equations

$$
W(X)=Z ; \quad W_{k}(X)=Z
$$

have, for $k$ big enough, either no solution or a unique solution in a neighborhood of $X^{*}$. These two equations are equivalent, respectively, to

$$
\phi_{k}(X)=X, \quad \phi(X)=X
$$

where

$$
\phi_{k}(X):=X+A_{k}\left(Z-W_{k}(X)\right) ; \quad \phi(X):=X+A(Z-W(X)) .
$$

Therefore, the result will be proved if we show that $\phi_{k}$ and $\phi$ are strict contractions in some neighborhood of $X^{*}$.

Since

$$
D \phi(X)=I-A D W(X)=A\left(D W\left(X^{*}\right)-D W(X)\right)
$$

we have from (A7) that

$$
X \in \bar{B}\left(X^{*} ; \eta\right) \Rightarrow\|D \phi(X)\| \leq \frac{1}{3}
$$

which proves that $\phi$ is a strict contraction in $\bar{B}\left(X^{*} ; \eta\right)$.

On the other hand,

$$
D \phi_{k}(X)=I-A_{k} D W_{k}(X)=A_{k}\left(D W_{k}\left(X^{*}\right)-D W_{k}(X)\right)
$$

so that taking into account (A6), we obtain for $k \geq k_{2}, k \in I$,

$$
\left\|D \phi_{k}(X)\right\| \leq 2\|A\|\left\|D W_{k}\left(X^{*}\right)-D W_{k}(X)\right\| .
$$

If $X \in \bar{B}\left(X^{*} ; r^{*}\right)$, we have

$$
\left\|D W_{k}(X)-D W_{k}\left(X^{*}\right)\right\| \leq 2 \sup _{X \in \bar{B}\left(X^{*} ; r^{*}\right)}\left\|D W_{k}(X)-D W(X)\right\|+\left\|D W(X)-D W\left(X^{*}\right)\right\| .
$$

Let $\hat{r}:=\min \left(\eta, r^{*}\right)>0$. Taking into account (A7) and (A8) we have for $k \geq k_{3}, k \in I$,

$$
\sup _{X \in \bar{B}\left(X^{*} ; \hat{r}\right)}\left\|D W_{k}\left(X^{*}\right)-D W_{k}(X)\right\| \leq \frac{1}{12\|A\|}+\frac{1}{3\|A\|}=\frac{5}{12\|A\|}
$$


and finally from (A9), choosing $\hat{k}:=\max \left(k_{2}, k_{3}\right)$, we have for $k \geq \hat{k}, k \in I$,

$$
\sup _{X \in \bar{B}\left(X^{*} ; \hat{r}\right)}\left\|D \phi_{k}(X)\right\| \leq 2\|A\| \sup _{X \in \bar{B}\left(X^{*} ; \hat{r}\right)}\left\|D W_{k}\left(X^{*}\right)-D W_{k}(X)\right\| \leq 2\|A\| \frac{5}{12\|A\|}=\frac{5}{6}<1
$$

which proves that $\phi_{k}$ is a strict contraction in $\bar{B}\left(X^{*} ; \hat{r}\right)$ and concludes the proof.

Proof of Theorem 3.5. Since the nonzero eigenvalues of matrices $D \bar{H}^{m}\left(Y^{*}\right)$ and $D H^{m}\left(X^{*}\right)$ coincide (Proposition 3.4), 1 is not an eigenvalue of $D H^{m}\left(X^{*}\right)$ and therefore we can apply Lemmas A. 1 and A.2, so there exist $r_{0}>0, k_{0} \in I$, such that for all $k \geq k_{0}, k \in I$ the mappings $W$ and $W_{k}$ defined in (A5) are one-to-one in $\bar{B}:=\bar{B}\left(X^{*} ; r_{0}\right)$ and moreover, for $s=1, \ldots, m, \lim _{k \rightarrow \infty} H_{k}^{s}=H^{s}$ and $\lim _{k \rightarrow \infty} D H_{k}^{s}=D H^{s}$ being both limits uniform in $\bar{B}$.

(a) We have to prove that for each $k \geq k_{0}, k \in I$, there exists a unique $X_{k}^{*} \in \bar{B}$ such that $X_{k}^{*}=$ $H_{k}^{m}\left(X_{k}^{*}\right)$ or, equivalently, $W_{k}\left(X_{k}^{*}\right)=0$.

The uniqueness is an immediate consequence of the fact that $W_{k}$ is one to one in $\bar{B}$.

Regarding the existence, let us recall that if a function $f$ is continuous and one-to-one in a compact set $C$ we have $f(\partial C)=\partial f(C)$ and int $f(C)=f($ int $C)$ where $\partial$ and int denote boundary and interior respectively.

We will prove the existence of $X_{k}^{*}$ by contradiction. If $X_{k}^{*}$ does not exist, then there exists a sequence $\left\{k_{n}\right\}_{n=1,2, \ldots} \subset I$ such that $\lim _{n \rightarrow \infty} k_{n}=+\infty$ and $\forall X \in \bar{B}, W_{k_{n}}(X) \neq 0$, i.e. $0 \notin W_{k_{n}}(\bar{B})$. Let us notice that $X^{*} \in \operatorname{int}(W(\bar{B}))$, which implies that $W\left(X^{*}\right)=0 \in \operatorname{int}(W(\bar{B}))$ and also $Z_{n}^{*}:=W_{k_{n}}\left(X^{*}\right) \in \operatorname{int}\left(W_{k_{n}}(\bar{B})\right)$.

Let $S_{n}$ be the line segment that joins 0 and $Z_{n}^{*}$. Obviously $S_{n} \cap \partial\left(W_{k_{n}}(\bar{B})\right) \neq \emptyset$, which allows us to chose $Z_{n} \in S_{n} \cap \partial\left(W_{k_{n}}(\bar{B})\right)$. Since $\lim _{n \rightarrow \infty} W_{k_{n}}=W$ uniformly in $\bar{B}$, then

$$
\lim _{n \rightarrow \infty} Z_{n}^{*}=\lim _{n \rightarrow \infty} W_{k_{n}}\left(X^{*}\right)=W\left(X^{*}\right)=0
$$

which, in turn, implies that $\lim _{n \rightarrow \infty} Z_{n}=0$.

This contradicts the fact that $Z_{n} \in \partial\left(W_{k_{n}}(\bar{B})\right)$. Indeed, since $0 \in \operatorname{int}(W(\bar{B}))$ we have $d_{0}:=\operatorname{dist}(0, \partial(W(\bar{B})))>0$. Let us consider the set

$$
E:=\left\{X \in \mathbb{R}^{N} ; \operatorname{dist}(X, \partial(W(\bar{B})))<\frac{d_{0}}{2}\right\}
$$

There exists a positive integer $n_{0}$ such that $\forall n \geq n_{0}, \partial\left(W_{k_{n}}(\bar{B})\right) \subset E$ and therefore $\left\|Z_{n}\right\|>d_{0} / 2$ so $\lim _{n \rightarrow \infty} Z_{n} \neq 0$.

In order to prove (a) it only remains to be shown that the period of $X_{k}^{*}$ is exactly $m$, what we will do after proving (b).

(b) We know from (a) that for each $k \geq k_{0}, k \in I, X_{k}^{*} \in \bar{B}$ and so $\left\{X_{k}^{*}\right\}_{k \in I}$ must have at least an accumulation point $Z^{*} \in \bar{B}$. We will prove that $Z^{*}$ is the unique accumulation point of $\left\{X_{k}^{*}\right\}_{k \in I}$ which implies that $\lim _{k \rightarrow \infty} X_{k}^{*}=Z^{*}$.

Let $\left\{X_{k_{n}}^{*}\right\}$ be a subsequence of $\left\{X_{k}^{*}\right\}_{k \in I}$ that converges to $Z^{*}$. Bearing in mind the continuity of $H^{m}$ and the fact that $H_{k_{n}}^{m}$ converges to $H^{m}$ uniformly in $\bar{B}$, straightforward calculations show that $\lim _{k_{n} \rightarrow \infty} H_{k_{n}}^{m}\left(X_{k_{n}}^{*}\right)=H^{m}\left(Z^{*}\right)$. Then,

$$
H^{m}\left(Z^{*}\right)=\lim _{k_{n} \rightarrow \infty} H_{k_{n}}^{m}\left(X_{k_{n}}^{*}\right)=\lim _{k_{n} \rightarrow \infty} X_{k_{n}}^{*}=Z^{*} .
$$

But since $W$ is one-to-one in $\bar{B}, H^{m}$ can only have one fixed point in $\bar{B}$ and therefore $Z^{*}$ is the unique accumulation point of $\left\{X_{k}^{*}\right\}_{k \in I}$. Moreover, since $X^{*} \in \bar{B}$ is a fixed point of $H^{m}$ it must be $Z^{*}=X^{*}$ and (b) is proved.

Now, let us go back to (a) and prove that the period of each $X_{k}^{*}$ is $m$. Indeed if this is not the case, there exists $s<m$ and a subsequence $\left\{X_{k_{n}}^{*}\right\}$ of $\left\{X_{k}^{*}\right\}_{k \in I}$ such that $H_{k_{n}}^{s}\left(X_{k_{n}}^{*}\right)=X_{k_{n}}^{*}$. 
Following a similar argument to the previous one, we can show that

$$
\lim _{k_{n} \rightarrow \infty} H_{k_{n}}^{s}\left(X_{k_{n}}^{*}\right)=H^{s}\left(X^{*}\right)
$$

and therefore

$$
X^{*}=\lim _{k_{n} \rightarrow \infty} X_{k_{n}}^{*}=\lim _{k_{n} \rightarrow \infty} H_{k_{n}}^{s}\left(X_{k_{n}}^{*}\right)=H^{s}\left(X^{*}\right)
$$

which contradicts the assumption of $X *$ being a $m$-periodic point.

(c) The result is an immediate consequence of the following fact: For each $\varepsilon \geq 0$, there exist $k_{0}^{*}(\varepsilon) \in I$ such that

$$
\forall k \geq k_{0}^{*}(\varepsilon), k \in I, \quad\left|\rho\left(D H_{k}^{m}\left(X_{k}^{*}\right)\right)-\rho\left(D \bar{H}^{m}\left(Y^{*}\right)\right)\right| \leq \varepsilon .
$$

This is turn follows from the facts that the nonzero eigenvalues of matrices $D \bar{H}^{m}\left(Y^{*}\right)$ and $D H^{m}\left(X^{*}\right)$ coincide, the eigenvalues of a matrix depend continuously of its entries and that $\lim _{k \rightarrow \infty} D H_{k}^{m}\left(X_{k}^{*}\right)=D H^{m}\left(X^{*}\right)$. This last equality is straightforward from the uniform convergence of $D H_{k}^{m}$ to $D H^{m}$ in $\bar{B}$, the continuity of $D H^{m}$ and $\lim _{k \rightarrow \infty} X_{k}^{*}=X^{*}$.

(d) Since $Y^{*}$ is hyperbolic and A.S., there exists $\gamma_{1} \in(0,1)$ such that $\rho\left(D \bar{H}^{m}\left(Y^{*}\right)\right) \leq \gamma_{1}<1$ and therefore, there exists a consistent matrix norm such that $\left\|D H^{m}\left(X^{*}\right)\right\| \leq \gamma_{1}$. By continuity, there exists $r_{1}, 0<r_{1}<r^{*}$ such that

$$
\sup _{X \in \bar{B}\left(X^{*} ; r_{1}\right)}\left\|D H^{m}(X)\right\| \leq \gamma_{1} .
$$

Moreover, since $\lim _{k \rightarrow \infty} X_{k}^{*}=X^{*}$ there exist $r_{2}>0$ and $k_{1} \in I$ such that for all $k>k_{1}, k \in I$, we have $\bar{B}\left(X_{k}^{*} ; r_{2}\right) \subset \bar{B}\left(X^{*} ; r_{1}\right)$ together with $X^{*} \in \bar{B}\left(X_{k}^{*} ; r_{2}\right)$.

Let us now show that for $k$ big enough, $H_{k}^{m}$ is a strict contraction in $\bar{B}\left(X_{k}^{*} ; r_{2}\right)$. Since, $\lim _{k \rightarrow \infty} D H_{k}^{m}(X)=D H^{m}$ uniformly in $\bar{B}\left(X^{*} ; r^{*}\right)$, for each $\varepsilon>0$ there exists $k(\varepsilon) \in I$ such that

$$
\forall k \geq k(\varepsilon), \quad k \in I, \sup _{X \in \bar{B}\left(X^{*} ; r^{*}\right)}\left\|D H_{k}^{m}(X)-D H^{m}(X)\right\| \leq \varepsilon .
$$

That is, if we fix $\gamma_{2}$ such that $0<\gamma_{1}<\gamma_{2}<1$, choosing $\varepsilon=\gamma_{2}-\gamma_{2}$ it follows that for all $k \geq k_{2}:=\max \left(k_{1}, k(\varepsilon)\right)$,

$$
\begin{aligned}
\sup _{X \in \bar{B}\left(X^{*} ; r_{1}\right)}\left\|D H_{k}^{m}(X)\right\| \leq \sup _{X \in \bar{B}\left(X^{*} ; r^{*}\right)}\left\|D H_{k}^{m}(X)-D H^{m}(X)\right\| \\
+\sup _{X \in \bar{B}\left(X^{*} ; r_{1}\right)}\left\|D H^{m}(X)\right\| \leq \varepsilon+\gamma_{1}=\gamma_{2}<1 .
\end{aligned}
$$

Therefore for $k \geq k_{2}, H_{k}^{m}$ is a strict contraction in $\bar{B}\left(X_{k}^{*} ; r_{2}\right)$ but since $X_{k}^{*}$ is the unique fixed point in $\bar{B}\left(X_{k}^{*} ; r_{2}\right)$, the orbit of $H_{k}^{m}$ starting in any initial condition in $\bar{B}\left(X_{k}^{*} ; r_{2}\right)$ must converge to $X_{k}^{*}$.

In order to prove (d) we only have to show that there exists a positive integer $n_{0}$ such that for $k$ big enough, $H_{k}^{m n_{0}}\left(X_{0}\right) \in \bar{B}\left(X_{k}^{*} ; r_{2}\right)$ which implies that $\lim _{n \rightarrow \infty} H_{k}^{m n}\left(X_{0}\right)=X_{k}^{*}$.

Let us begin by noting that $\lim _{n \rightarrow \infty} \bar{H}^{m n-1}\left(Y_{0}\right)=Y^{*}$ or, using, (A1), $\lim _{n \rightarrow \infty} H^{m n}\left(X_{0}\right)=X^{*}$, which assures the existence a positive integer $n_{0}$ such that

$$
\left\|H^{m n_{0}}\left(X_{0}\right)-X^{*}\right\| \leq \frac{r_{2}}{3} .
$$

Also, $\lim _{k \rightarrow \infty} X_{k}^{*}=X^{*}$ ensures that there exists $k_{3} \in I$ such that

$$
\forall k \geq k_{3}, \quad k \in I, \quad\left\|X_{k}^{*}-X^{*}\right\| \leq \frac{r_{2}}{3} .
$$


Finally, Hypothesis 3.7 allows us to apply Lemma A.1 in the point $X_{0}$, so there exists $\hat{r}_{0}>0$ such that $\lim _{k \rightarrow \infty} H_{k}^{m n_{0}}=H^{m n_{0}}$ uniformly in $\bar{B}\left(X_{0} ; \hat{r}\right)$ and therefore there exists $k_{4} \in I$ such that

$$
\forall k \geq k_{4}, k \in I, \sup _{X \in \bar{B}\left(X_{0} ; \hat{r}\right)}\left\|H_{k}^{m r_{0}}\left(X_{0}\right)-H^{m n_{0}}\left(X_{0}\right)\right\| \leq \frac{r_{2}}{3} .
$$

Choosing $k_{0}^{* *}:=\max \left(k_{2}, k_{3}, k_{4}\right)$ we have for all $k \geq k_{0}^{* *}, k \in I$,

$$
\left\|H_{k}^{m n_{0}}\left(X_{0}\right)-X_{k}^{*}\right\| \leq\left\|H_{k}^{m n_{0}}\left(X_{0}\right)-H^{m n_{0}}\left(X_{0}\right)\right\|+\left\|H_{k}^{m n_{0}}\left(X_{0}\right)-X^{*}\right\|+\left\|X^{*}-X_{k}\right\| \leq r_{2}
$$

and hence $H_{k}^{m n_{0}}\left(X_{0}\right) \in \bar{B}\left(X_{k}^{*} ; r_{2}\right)$. 